\title{
Hydroxyl Radical
}

National Cancer Institute

\section{Source}

National Cancer Institute. Hydroxyl Radical. NCI Thesaurus. Code C1130.

A monovalent radical consisting of one hydrogen and oxygen atom found in bases,

carboxylic acids, and alcohols. 